\title{
Propiedades del suelo afectadas por el tiempo de descanso en un sistema de roza-tumba-quema
}

\section{Soil characteristics due to resting time in a slash and burn system}

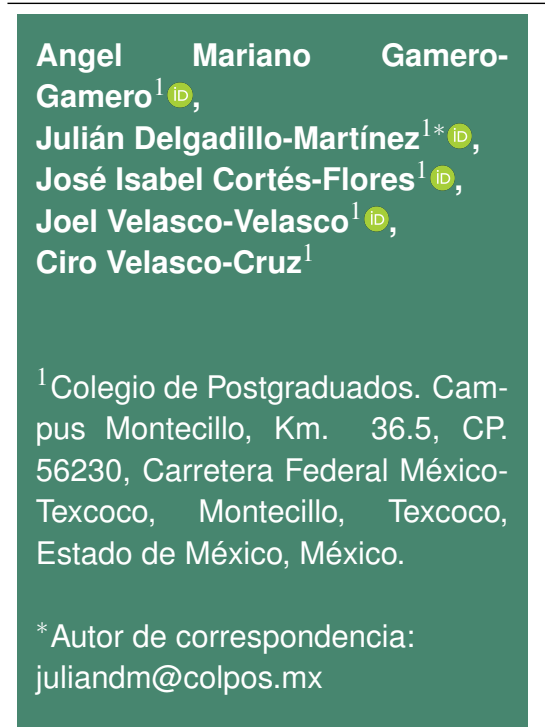

Artículo científico

Recibido: 21 de enero 2019

Aceptado: 03 de abril de 2020

Como citar: Gamero-Gamero AM, Delgadillo-Martínez J, Cortés-Flores Jl, VelascoVelasco J, Velasco-Cruz C (2020) Propiedades del suelo afectadas por el tiempo de descanso en un sistema de roza-tumbaquema. Ecosistemas y Recursos Agropecuarios 7(1): e2098. DOI: 10.19136/era.a7n1.2098

RESUMEN. El estudio de los sistemas agrícolas tradicionales representa una opción ante la necesidad de conservar los ecosistemas naturales e incrementar la producción de alimentos. Una mejor comprensión de la dinámica ecológica de la roza-tumba-quema (RTQ) puede permitir la toma de decisiones encaminadas al manejo racional de los ecosistemas donde esta se practica. Por lo anterior, el objetivo fue describir la relación de las comunidades microbianas con la materia orgánica y la condición nutrimental del suelo. Se calcularon los índices de diversidad de Shannon $\left(H^{\prime}\right)$ a partir del perfil metabólico de las poblaciones con el uso de placas BIOLOG-ECO ${ }^{\mathrm{TM}}$, el contenido de materia orgánica (MO), nitrógeno total, nitratos, amonio, fósforo extractable, textura, densidad aparente, capacidad de campo y punto de marchitez permanente del suelo. Las propiedades físicas no fueron significativamente diferentes. El pH, contenido de $\mathrm{MO}$ y de nitratos se modificó con el tiempo de descanso. No se encontró relación entre la diversidad microbiana $\left(H^{\prime}\right)$ y el tiempo de descanso $(t)$, pero sí diferencias significativas $(p$ $<0.05)$ entre las parcelas con mayor y menor tiempo de descanso, siendo más diversa esta última.

Palabras clave: Bacterias heterotróficas, bosque tropical caducifolio, índice de diversidad de Shannon, diversidad funcional, Tierra Caliente.

ABSTRACT. The study of traditional agricultural systems performs an option for natural-ecosystems conservation and increase crop production. The slashand-burn system (RTQ) has been declared obsolete and harmful, but this outlook is inconclusive. The aim of this study is to promote a better understanding of its ecological dynamics, which can guide for take decisions of the rational management of the ecosystems where it is located. A fallow chronosequence of the RTQ was analyzed in a tropical deciduous forest on leptosols, with the objective of describing the relationship of the microbial communities with the fallow period and soil organic matter. It is reported the heterotrophic bacteria functional diversity (Shannon index - $\left.\mathrm{H}^{\prime}\right)$ and its activity, since metabolic profile of the microbial populations using BIOLOG-ECO ${ }^{T M}$ plates, organic matter content, total nitrogen, nitrate, ammonium, extractable phosphorus, texture, bulk density, field capacity and permanent wilting point. The soil physical properties were similar at all over plots. Fallow time affected $\mathrm{pH}$, organic-matter ad nitrates content. No clear relation was found between $\mathrm{H}^{\prime}$ and the fallow time (t) but there were $H^{\prime}$ significant differences $(p<0.05)$ between higher and less fallow time.

Key words: Heterotrophic bacteria, tropical dry forest, Shannon diversity index, functional diversity, Tierra Caliente. 


\section{INTRODUCCIÓN}

La roza-tumba-quema (RTQ) es un sistema itinerante de cultivo fundamentado en alternar el uso intensivo de un terreno con periodos largos de descanso. Esta técnica, consiste en derribar una sección de bosque maduro, extraer la parte maderable y la leña, dejar secar y luego quemar el resto del material vegetativo. Sobre el terreno se siembra de uno a tres ciclos anuales (Pérez-García y del Castillo 2016). Luego se permite la recuperación de la vegetación, con o sin intervención humana, durante varios años (Riveiro-Filho et al. 2013). El conocimiento de los recursos locales proporciona la base para el funcionamiento de este sistema y su racionalidad se sustenta en los descansos largos y el policultivo (Toledo et al. 2008). Esta técnica se practica en la zona tropical del mundo desde hace aproximadamente diez mil años, aunque históricamente, no se limita a estas latitudes (Tomson et al. 2016).

El cambio de uso de tierra mediante esta técnica, en condiciones de trópico seco, modifica el ciclo de nutrientes (Amorim et al. 2014), y presenta mayor disponibilidad de estos después de la quema (Giardina et al. 2000a); aunque hay evidencia de que estos incrementos son significativos después del primer mes del fuego (Singh et al. 2017). Con frecuencia, el carbono lábil resultante de la quema incrementa la actividad microbiológica, misma que facilita múltiples procesos en el ambiente edáfico (Giardina et al. 2000b). Al respecto, Riveiro-Filho et al. (2013) indican que el fuego y los descansos cortos impactan de forma negativa en el componente biológico y que el aspecto crítico para que el sistema se sostenga es el periodo de descanso. La calidad del suelo es la capacidad que tiene para cumplir con sus funciones dentro de límites establecidos, permitiendo la limpieza del agua y el aire, así como la salud y sustento de animales, plantas y seres humanos (Karlen et al. 1997). La cual se estima mediante diversos parámetros, como el carbono lábil (Ginebra et al. 2015), el carbono de la biomasa microbiana (Fregoso 2008), las propiedades físicas y químicas (Jaurixje et al. 2013), la mesofauna (Socarrás 2013), la actividad enzimática (Ochoa et al. 2007), entre otras. Las comunidades microbianas constituyen un aspecto importante en la calidad del suelo (Jackson et al. 2003). Los microorganismos son responsables de procesos fisiológicos y metabólicos edáficos (Bastida et al. 2008); además de que presentan una rápida respuesta a los cambios en el manejo del suelo, en su condición ambiental o contaminación (Ferreras et al. 2009, Pagano et al. 2011). Las propiedades físicas y químicas del suelo, son más fácil de medir (Di Ciocco et al. 2014), y están asociados a los cambios biogeoquímicos del ecosistema (Jackson et al. 2003) y se reconocen como buenos indicadores de la calidad del suelo (Doran y Zeiss 2000, Ochoa et al. 2007).

En la región de Tierra Caliente de Michoacán, con bosque tropical caducifolio (Rzedowski 2006), se practica la RTQ desde tiempos prehispánicos, para la producción de maíz (Rojas 1991). En los últimos 50 años se ha incorporado la fertilización y el uso de herbicidas, además de la presencia histórica y generalizada de la ganadería (Cochet 2001); por lo que la conversión temporal en potrero reduce el periodo de descanso. Dadas las condiciones para la producción de maíz en esta región, se desconoce si las comunidades microbianas son un indicador adecuado para valorar la calidad de los suelos, de igual manera la relación entre la diversidad funcional microbiana y el periodo de descanso, y si esta diversidad se relaciona con la materia orgánica. Por lo anterior, el objetivo fue describir la influencia que tiene el tiempo de descanso sobre las comunidades microbianas al nivel de diversidad funcional, la materia orgánica y la condición macro nutrimental del suelo en un ambiente de bosque tropical caducifolio, para conocer su papel en la recuperación de la calidad del suelo y su utilidad como indicadores de calidad en estas condiciones.

\section{MATERIALES Y MÉTODOS}

\section{Ubicación}

El área de estudio se encuentra en el ejido Acatén, en la parte suroeste del municipio de Madero, en la región Tierra Caliente de Michoacán, México. La vegetación original fue bosque tropical caducifolio 
(Rzedowski 2006) y el clima dominante es cálido subhúmedo con lluvias en verano $\left(A_{0}\right)$ (García 2004). De acuerdo con el Instituto Nacional de Estadística, Geografía e Informática y con la verificación en campo, el tipo de suelo dominante es leptosol, lo que indica que son suelos jóvenes y someros de origen volcánico (Gómez-Vasconcelos et al. 2015).

\section{Identificación de los sitios de muestreo}

Se identificaron dos sitios que conformaron, en conjunto, una cronosecuencia de descanso después de la RTQ, para tener la opción de conocer los cambios temporales en la composición de las comunidades sin que el tiempo requerido fuera necesariamente prolongado (Smith et al. 2015).

El primer sitio se localiza a $19^{\circ} 16^{\prime} 15^{\prime \prime}$ LN y $101^{\circ} 17^{\prime} 46^{\prime \prime}$ LO, a una altitud de $1660 \mathrm{~m}$, corresponde a una parcela con vegetación secundaria que tiene más de 30 años sin manejo, identificada como $\mathrm{A}$, con un área aproximada de $1680 \mathrm{~m}^{2}$, orientación oeste y pendiente media de $64.5 \%$. El segundo sitio se encuentra aproximadamente a $2.3 \mathrm{~km}$ al noroeste del primero, se localiza $19^{\circ} 17^{\prime} 22$ " LN y $101^{\circ} 18^{\prime} 18^{\prime \prime}$ LO y altitud de $1685 \mathrm{~m}$; es una parcela segmentada, donde cada sección tiene distinto tiempo de uso o descanso después de la RTQ. Tiene un área total aproximada de $6754.4 \mathrm{~m}^{2}$, orientación suroeste, con pendiente dominante homogénea de $62.9 \%$. Las parcelas se identificaron como B, C y $D$. En $B$ han pasado tres años después de la RTQ, correspondientes a dos años de cultivo y uno de descanso; en $C$ han pasado cinco años después de la $R T Q$, de los cuales dos fueron de cultivo y tres de descanso; y $D$ tiene ocho años desde que se realizó la RTQ, dos de ellos de cultivo y los otros seis de descanso.

\section{Muestreo de suelo}

Se realizó un muestreo sistemático en abril de 2017, con nueve puntos en cada parcela, con distribución equitativa con respecto a la pendiente. De cada punto se tomó una muestra simple con pala recta, a profundidad de 0 a $15 \mathrm{~cm}$, la cual se almacenó a temperatura ambiente durante una sema- na. Las muestras se secaron a temperatura ambiente, se tamizaron en malla calibre 10 y de tres muestras se obtuvo una muestra compuesta.

\section{Análisis físico y químico del suelo}

Las características físicas determinadas fueron la densidad aparente (Dap) por el método del terrón parafinado; textura, por el método de Bouyoucos (Arteta 2001) modificado, separando las arenas a través de un tamiz calibre $30(0.595 \mathrm{~mm})$. Capacidad de campo (CC) y punto de marchitez permanente (PMP), por medio de membranas de presión; y el contenido de humedad. También se determinó el contenido de materia orgánica total (MO) por el método de Walkley y Black, el pH en una solución 1:2 de suelo/agua, $\mathrm{N}$ total por el método de Kjeldhal; nitratos (Singh 1988), amonio (Baethgen y Alley 2008), y fósforo extraíble por el método de Bray y Kurtz (1945).

Para el estudio de las comunidades bacterianas heterotróficas se utilizó el enfoque de perfiles fisiológicos a nivel de comunidad (CLPP), construidos a partir del desarrollo de color durante la incubación de muestras diluidas de suelo en placas BIOLOG-ECOPLATE ${ }^{\text {TM }}$ por triplicado para cada sitio, con un total de 12 placas.

Esta técnica de placas se basa en la reducción de tetrazolium y su cambio de incoloro a violeta, a partir del uso de distintas fuentes de C (Garland y Mills 1991, Kaffe-Abramovich y Steinberger 2006). Para preparar las placas, primero se pesaron 3.33 g de cada muestra compuesta, que se agregaron a frascos con $30 \mathrm{ml}$ de agua destilada, previamente esterilizada, para luego mezclar y agitar a $150 \mathrm{rpm}$ durante 30 minutos.

Se realizaron diluciones hasta $10^{-3}$, de esta última se tomaron $150 \mu \mathrm{L}$ para inocular cada uno de los 96 pozos de la placa BIOLOG-ECOPLATE ${ }^{\text {TM }}$, con 31 fuentes distintas de carbono por triplicado, y tres pozos con agua destilada como testigo. La actividad metabólica microbiana se calculó con base en seis grupos de fuentes de C: 1) ácidos carboxílicos, 2) aminas y amidas, 3) aminoácidos, 4) carbohidratos, 5) misceláneos y 6) polímeros. 
Las placas se incubaron a $28{ }^{\circ} \mathrm{C}$ durante 96 $\mathrm{h}$, pero se realizaron lecturas cada $24 \mathrm{~h}$ en espectrofotómetro Synergy2 (Biotek), a longitud de onda de $590 \mathrm{~nm}$. El análisis de los datos se realizó con el uso del software Gen5 Microplate Reader (Biotek, Versión 5.1). El promedio de la respuesta metabólica (RM), también llamado desarrollo promedio de color (AWCD), se calculó por fuente de carbono mediante la fórmula:

$$
A W C D=\sum_{i=1}^{S} \frac{\left(D O_{i}-D O_{c}\right)}{n}
$$

Donde: $\mathrm{DO}_{i}$ es la densidad óptica promedio de la muestra, $\mathrm{DO}_{c}$ la densidad óptica promedio del control (agua destilada), y $\mathrm{n}$ el número de fuentes de carbono.

Las $\mathrm{DO}_{i}$ se utilizaron para obtener la abundancia relativa por grupo funcional ( $p i)$ con la fórmula:

$$
p_{i}=\frac{n_{i}}{N}
$$

Donde: $\mathrm{n}_{i}$ es la DO de la fuente de carbono i y $\mathrm{N}$ es la sumatoria de todas las $\mathrm{DO}_{i}$.

A partir de estos datos, se calculó el índice de Shannon $\left(H^{\prime}\right)$ de la fórmula:

$$
H^{\prime}=-\sum_{i=1}^{S} p_{i} \ln p_{i}
$$

Donde: $\mathrm{S}$ es el número de fuentes de $\mathrm{C}$ por tipo de actividad metabólica. Para ácidos carboxílicos es nueve, aminas y amidas dos, aminoácidos seis, carbohidratos siete, misceláneos tres y polímeros cuatro.

Se partió de la medición indirecta de la actividad metabólica por medio del desarrollo de color en la placa (AWCD), para obtener un índice de diversidad de Shannon $\left(\mathrm{H}^{\prime}\right)$ para cada parcela. Para calcular los índices de Shannon ( $\left.H^{\prime}\right)$ se consideraron las lecturas realizadas $96 \mathrm{~h}$ después de la inoculación, que corresponden a un desarrollo de color más estabilizado. Se realizó el conteo, en cajas Petri, de las unidades formadoras de colonias (UFC) presentes en el suelo de las parcelas. Se prepararon los medios de cultivo de agar nutritivo (AN), Rennie (R) y papadextrosa-agar con rosa de bengala (PDA), para bacterias totales $(\mathrm{Bt})$, bacterias fijadoras de nitrógeno (Bfn) y hongos totales $(\mathrm{Ht})$, respectivamente.
Se prepararon tres muestras compuestas de suelo por cada parcela. Por cada muestra se realizó una dilución de $10^{-1}$ en agua destilada estéril, la cual se agitó durante $30 \mathrm{~min}$ a $150 \mathrm{rpm}$. Posteriormente, se realizaron las diluciones de $10^{-2}$ a $10^{-6}$ de cada muestra. Se inocularon cajas Petri, por triplicado, con $1 \mathrm{ml}$ de las diluciones $10^{-5}$ y $10^{-6}$ para Bt, $10^{-3}$ y $10^{-4}$ para Bfn y $10^{-2}$ y $10^{-3}$ para Ht. Estas se incubaron a $28^{\circ} \mathrm{C}$ durante $24 \mathrm{~h}$, para luego cuantificar de forma visual las UFC.

\section{Análisis estadísticos}

Los análisis se realizaron con el software libre de R-project. Para verificar la normalidad de los datos se empleó la prueba de chi cuadrada a partir del comando pearson.test de la librería nortets. Para conocer la existencia de diferencias entre las medias de las variables MO, FL, FP, H' y tiempo de descanso en años (t), se realizaron análisis de varianzas en el software libre de R-project utilizando el comando aov(), posteriormente se aplicó la prueba de Tukey con el comando HSD.test() de la librería agricolae con $\alpha=0.05$.

\section{RESULTADOS}

\section{Propiedades físicas y químicas}

Las variables físicas (Dap, CC, PMP, humedad aprovechable) no presentaron diferencias significativas $(p<0.05)$ con respecto al tiempo de descanso del terreno. En todos los sitios hubo un porcentaje bajo de humedad aprovechable, debido a la poca diferencia entre la CC y el PMP (Tabla 1). Los contenidos de nitrógeno total, amonio y fósforo extraíble no presentaron diferencias significativas $(p<0.05)$ con relación al tiempo de descanso. El contenido de nitrato fue mayor en suelo con descanso de más de 30 años (Tabla 2).

La concentración de fósforo extraíble, nitrato y amonio presentó valores bajos. Al observar el comportamiento de estos nutrimentos a través del tiempo de descanso, se observó un decremento notorio en la parcela con un año de descanso (C), con respecto a la parcela con tres años de descanso (B) y posteriormente un nuevo incremento en la parcela 
Tabla 1. Propiedades físicas evaluadas con respecto al tiempo de descanso en suelos leptosoles donde la vegetación original fue bosque tropical caducifolio.

\begin{tabular}{|c|c|c|c|c|c|c|c|}
\hline $\begin{array}{l}\text { Tiempo después } \\
\text { de la quema } \\
\text { (años) }\end{array}$ & $\begin{array}{l}\text { Tiempo de } \\
\text { descanso } \\
\text { (años) }\end{array}$ & $\begin{array}{c}\text { Textura } \\
\text { dominante }\end{array}$ & $\begin{array}{c}\text { Capacidad } \\
\text { de campo } \\
\text { (CC \%) }\end{array}$ & $\begin{array}{l}\text { Punto de mar- } \\
\text { chitez perma- } \\
\text { nente (PMP \%) }\end{array}$ & $\begin{array}{c}\text { Humedad } \\
\text { aprovechable } \\
\text { (CC-PMP, \%) }\end{array}$ & $\begin{array}{l}\text { Humedad al } \\
\text { momento del } \\
\text { muestreo (\%) }\end{array}$ & $\begin{array}{c}\text { Densidad apa- } \\
\text { rente - Dap } \\
\left(\mathrm{Mg} \cdot \mathrm{m}^{-3}\right)\end{array}$ \\
\hline 3 & 1 & Franco limoso & 24.4 & 18.8 & 5.6 & 4.0 & 1.54 \\
\hline 5 & 3 & Arcillo limoso & 25.6 & 20.7 & 4.9 & 6.6 & 1.57 \\
\hline 8 & 6 & Franco & 22.1 & 16.9 & 5.2 & 3.9 & 1.58 \\
\hline$>30$ & $>30$ & Franco limoso & 25.5 & 20.6 & 4.9 & 7.8 & 1.52 \\
\hline
\end{tabular}

Tabla 2. Variables químicas del suelo con respecto al tiempo de descanso de la tierra.

\begin{tabular}{|c|c|c|c|c|c|c|c|}
\hline $\begin{array}{l}\text { Tiempo después de } \\
\text { la quema (años) }\end{array}$ & $\begin{array}{c}\text { Tiempo de } \\
\text { descanso (años) }\end{array}$ & $\begin{array}{l}\mathrm{Nt} \\
(\%)\end{array}$ & $\begin{array}{c}\mathrm{P} \\
\left(\mathrm{mg} \cdot \mathrm{kg}^{-1}\right)\end{array}$ & $\begin{array}{c}\mathrm{NO}_{3^{-}} \\
\left(\mathrm{mg} \cdot \mathrm{kg}^{-1}\right)\end{array}$ & $\begin{array}{c}\mathrm{NH}_{4+} \\
\left(\mathrm{mg} \cdot \mathrm{kg}^{-1}\right)\end{array}$ & $\begin{array}{l}\text { Contenido } \\
\text { de } \mathrm{MO}(\%)\end{array}$ & $\begin{array}{c}\text { Reacción del } \\
\text { suelo }(\mathrm{pH})\end{array}$ \\
\hline 3 & 1 & $0.16^{a}$ & $39.7^{a}$ & $46.7^{b}$ & $37.3^{a}$ & $4.3^{a b}$ & $6.05^{c}$ \\
\hline 5 & 3 & $0.15^{a}$ & $36.5^{a}$ & $28.0^{b}$ & $23.3^{a}$ & $4.5^{a b}$ & $6.43^{a b}$ \\
\hline 8 & 6 & $0.18^{a}$ & $36.1^{a}$ & $65.3^{a b}$ & $42.0^{a}$ & $3.3^{b}$ & $6.23^{b c}$ \\
\hline$>30$ & $>30$ & $0.18^{a}$ & $21.6^{a}$ & $144.7^{a}$ & $65.3^{a}$ & $6.1^{a}$ & $6.58^{a}$ \\
\hline
\end{tabular}

con seis años de descanso (D), presentando el valor mayor la parcela con más de 30 años de descanso (A).

La materia orgánica y el pH tuvieron una tendencia creciente en función del tiempo de descanso. Los índices de Shannon ( $\left.\mathrm{H}^{\prime}\right)$ presentaron diferencias significativas $(p<0.05)$ entre las condiciones más contrastantes, es decir, entre la parcela con un año de descanso y la parcela con más de 30 años de descanso ( $B$ y $A$, respectivamente); estos valores fueron, en general, elevados. El pH es un factor importante, ya que al ser un valor exponencial, indica grandes cambios en las características reactivas del suelo. El $\mathrm{pH}$ tuvo diferencias significativas para los sitios con más de 30 años de descanso (A) y con un año de descanso (B); fue más próximo a la neutralidad en la parcela más descansada $(A)$ y ligeramente ácido en el sitio más recientemente cultivado (B).

\section{Características microbiológicas}

La diversidad microbiana (índice H') presentó diferencias entre la parcela con 3 años de descanso que superó sólo al área natural con más de 30 años de descanso (Tabla 3). El consumo aminas/amidas, carbohidratos, misceláneos y polímeros no presentó diferencias significativas $(p<0.05)$ entre los distintos tiempos de descanso del terreno, mientras que el uso de ácidos carboxílicos fue significativamente distinto $(p<0.05)$ para la parcela con tres años de descanso y la que tiene más de 30 años de descanso, siendo más alto en la primera (Figura 1).

El análisis de varianza no mostró diferencias significativas $(p<0.05)$ en el tamaño de la población de Bt, Bfn y Ht para todos los tiempos de descanso (Tabla 3). La cantidad de UFC de Bt fue mucho mayor que la de $\mathrm{Ht}$, mientras que las Bfn se mantuvieron en niveles altos, estando presentes en todas las parcelas $y$ sin diferencia significativa $(p<0.05)$ con respecto a Bt.

\section{DISCUSIÓN}

Los resultados de las variables físicas concuerdan con lo reportado por Medeiros et al. (2017), quienes tampoco observaron diferencias en variables físicas a una profundidad de 5 a $10 \mathrm{~cm}$ en estados de sucesión de 15, 30 y 50 años en un bosque tropical seco; pero, sí encontraron mejoras en las propiedades químicas con los tiempos de descanso más largos. Al respecto Alegre y Cassel (1996) indican que la RTQ no afecta la estructura y densidad aparente del suelo; aunque la quema deja expuesta una mayor superficie de suelo que favorece la erosión hídrica y eólica, sobre todo en los primeros años de cultivo (Dos Santos et al. 2017). Esta es la razón principal por la que se presentan grandes pérdidas de suelo y, por ende, nutrimentos después de la quema; lo que se ha documentado después de incen- 
Tabla 3. Presencia de UFC en parcelas con distinto tiempo de descanso después de la RTQ. Los conteos se realizaron en cajas Petri después de $24 \mathrm{~h}$ de inoculación.

\begin{tabular}{ccccc}
\hline $\begin{array}{c}\text { Tiempo de } \\
\text { descanso (años) }\end{array}$ & $\begin{array}{c}\text { Bacterias tota- } \\
\text { les (Bt) }\end{array}$ & $\begin{array}{c}\text { Bacterias fijadoras } \\
\text { de nitrógeno }(\mathrm{Bfn})\end{array}$ & $\begin{array}{c}\text { Hongos tota- } \\
\text { les }(\mathrm{Ht})\end{array}$ & $\begin{array}{c}\text { Índice de diversidad } \\
\text { de Shannon }\left(\mathrm{H}^{\prime}\right)\end{array}$ \\
\hline \multicolumn{3}{c}{ UFC $\times 104$} & & UFC $\times 102$ \\
3 & 38.9 & 42.2 & 12.33 & $3.32^{b c}$ \\
6 & 73.9 & 43.7 & 40.67 & $3.36^{a}$ \\
$>30$ & 60.0 & 64.3 & 43.00 & $3.34^{a b}$ \\
\hline Se muestran los valores promedio. Las letras denotan diferencia significativa entre los valores (Tukey, \\
$\alpha=0.05$ ).
\end{tabular}

\section{Actividad metabólica de las bacterias}

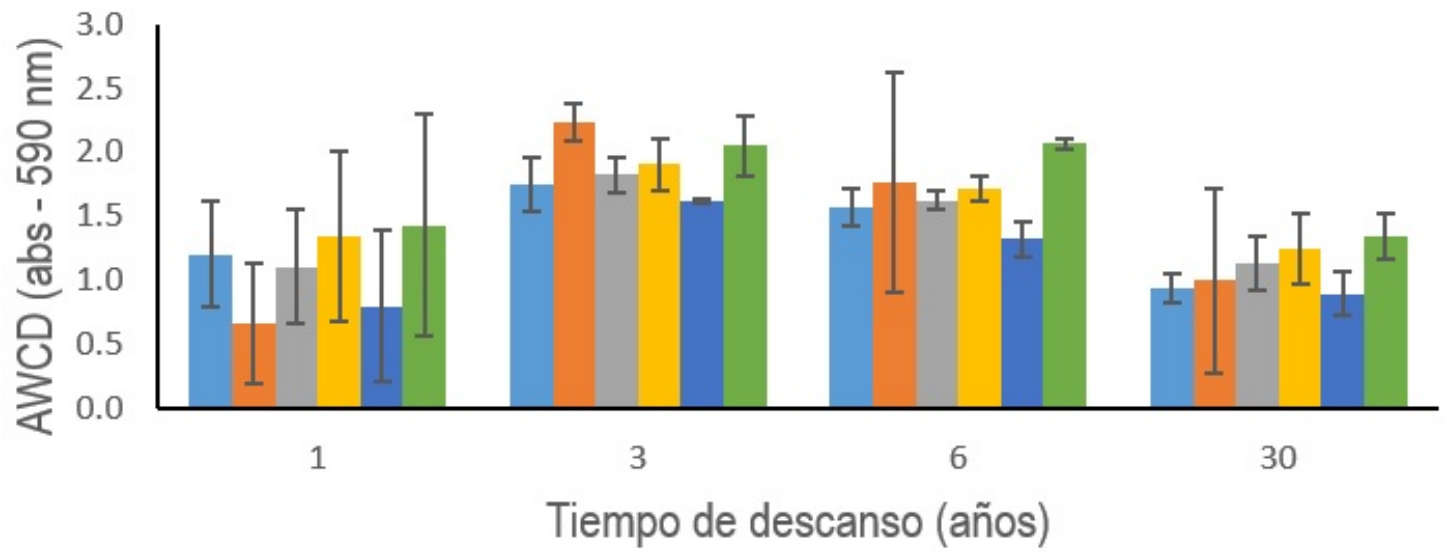

\section{Ácidos carboxílicos $\square$ Aminas/Amidas $\quad$ Aminoacidos \\ Carbohidratos a Misceláneos a Polimeros}

Figura 1. Actividad metabólica de las bacterias del suelo por fuente de carbono utilizada, se reportan las lecturas de absorbancia a las 96 h de incubación. Las barras representan la desviación estándar.

dios forestales (Giardina et al. 2000b). Los efectos se manifiestan con la pérdida de la cubierta vegetal, reducción de la infiltración y aumento en la erosión hídrica (Wilson et al. 2018, Fernández et al. 2019, Rodrigues et al. 2019). La baja humedad disponible en el suelo acentúa la fuerte estacionalidad de la zona con respecto a la precipitación (Rzedowski 2006), y es una restricción importante durante la temporada de crecimiento en estos suelos poco desarrollados y someros (Maass et al. 2005, Gómez-Vasconcelos et al. 2015). En los primeros años después de la quema se esperara un descenso de las formas disponibles de nitrógeno por la quema misma y por consecuencia de la erosión superficial. La quema incrementa la mineralización de las formas orgánicas de nitrógeno, pero las pérdidas por volatilización resultan en un balance negativo para este elemento (Giardina et al. 2000a), pero la fertilización practicada por los dueños de las parcelas compensa estas pérdidas.

La mayor presencia de $\mathrm{P}$ después de la quema, se puede explicar por el aumento de la temperatura del suelo, lo que favorece la mineralización del P orgánico (Giardina et al. 2000b) y libera los fosfatos ocluidos en los agregados del suelo (MataixSolera et al. 2011). Los efectos de la quema en el contenido de $\mathrm{P}$ disponible pueden ser drásticos en los primeros años, llegando a un equilibrio en años subsecuentes de descanso, principalmente provocados 
por el establecimiento de la vegetación natural y el restablecimiento de los ciclos biogeoquímicos (Moya et al. 2018). Los valores de pH contrastan con el hecho de que la ceniza, producida por la quema, tiende a alcalinizar el suelo (Giardina et al. 2000a, Urretavizcaya, 2010). Este efecto es inmediato al evento fuego y los suelos del sitio son de origen volcánico, más tendientes a la acidez (Gómez-Vasconcelos et al. 2015). No se encontró una correspondencia entre el tiempo de descanso y el contenido de $\mathrm{MO}$, ya que este último fue mayor en la parcela con más tiempo de descanso, con diferencia significativa $(p<0.05)$ solo con respecto al sitio con seis años de descanso. Al respecto, Medeiros et al. (2017) reportan un incremento significativo de carbono orgánico del suelo en los sitios con mayor tiempo de regeneración natural en un área tropical seca. Mientras que Kotto-Same et al. (1997) encontraron una fuerte correlación lineal positiva entre el tiempo de descanso y el carbono total del sistema en un área tropical húmeda.

La biomasa de la hojarasca en los bosques tropicales secos no es tanta, comparada con la producida en áreas de trópico húmedo, aun así, representa un reservorio significativo en el reciclaje del carbono y otros nutrientes (Amorim et al. 2014). No obstante, el suministro más importante de carbono proviene de la constante regeneración de las raíces finas, fenómeno que fue descrito por Castellanos et al. (2001), quienes señalan que el tiempo medio de residencia de las raíces finas es menor que el de otros residuos vegetales, incorporándose más pronto al suelo, llegando a tener aportes de biomasa significativos en condiciones naturales.

La constancia que mantuvo el índice de diversidad de Shannon ( $\left.\mathrm{H}^{\prime}\right)$ en los distintos tiempos de descanso confirma la resiliencia de las comunidades microbianas, que se puede explicar por su homeostasis estequiométrica (Spohn, 2016) y la posible redundancia de especies dentro de las poblaciones (Zamora et al. 2012). Comparados con reportes que indican menores índices de biodiversidad microbiana (Arteaga-Garibay et al. 2016, WingChing-Jones y Uribe 2016), los valores de H' fueron elevados en este trabajo, lo que indica que la diversidad biológica presente en los bosques tropicales y subtropicales, en los horizontes superficiales (Sharmah y Jha 2014).

En otros trabajos, hay evidencia de que la actividad bacteriana es estimulada por el suministro de materiales orgánicos en el suelo (Riveiro-Filho et al. 2013, Amorim et al. 2014, Kumar et al. 2017 Manjunath et al. 2018), sin embargo, y en contra de la hipótesis propuesta, la diversidad funcional bacteriana no estuvo correlacionada con el contenido de MO y sólo fue mayor con tres años de descanso en comparación con el sitio natural con más de 30 años de descanso. Lo anterior, indica capacidad de resiliencia entre las comunidades microbianas del suelo durante uno, tres y seis años después de la quema. Posiblemente, el manejo de las parcelas provoca activación de los microorganismos. Sin embargo, con periodos de descanso mayores a 30 años, los procesos biogeoquímicos se estabilizan y la biodiversidad microbiana se mantiene en tasas basales. En tamaño de la población, las comunidades microbianas no cambiaron debido a los diferentes periodos de descanso.

Se ha reportado mayor actividad microbiana en el suelo de la rizósfera de dos razas de maíz en la etapa inicial y final del cultivo, indicando que los hechos que pudieron incentivarla son: el contenido de carbono orgánico en los residuos de la cosecha anterior y la mayor producción de exudados radicales en las últimas semanas del ciclo (Arteaga-Garibay et al. 2016). Esto puede explicar por qué en la parcela con menos descanso, o más recientemente cultivada, el índice de diversidad mantiene un valor elevado y sin diferencia estadística con la que tiene más tiempo de descanso, dada la presencia reciente de maíz. En el caso de la parcela con tres años de descanso, aunque su contenido de $\mathrm{MO}$ no tuvo diferencia significativa con el contenido de $\mathrm{MO}$ de la parcela con menos tiempo de descanso, la diversidad funcional de bacterias ( $\left.H^{\prime}\right)$ sí la tuvo, presentando un valor más alto en la parcela con tres años de descanso, lo que confirma la independencia entre contenido de $\mathrm{MO}$ y $\mathrm{H}^{\prime}$ en el área de estudio. Al respecto, Li et al (2020) indican que la mayor diversidad microbiana del suelo se asocia a mayores aportes de $\mathrm{MO}$ (cobertura vegetal, incorporación de abonos o residuos vegetales) o por no manejar el suelo (labranza cero); por lo con- 
trario, en las condiciones de este estudio, el cambio en diversidad microbiana ( $\left.H^{\prime}\right)$ se asoció al manejo de la parcela y los cambios en la actividad microbiana del suelo; cómo aceleración de la mineralización de la MO, modificación de los diferentes tipos de sustratos disponibles. De manera similar, Li et al (2020) indicaron que en área naturales, periodos largos de sucesión vegetal traen consigo cambios benéficos en las propiedades del suelo, pero no necesariamente aumentos en la biodiversidad microbiana.

Se sabe que la presencia de vegetación aporta fuentes de carbono para los microorganismos edáficos (Revillini et al. 2016), por lo que la mayor diversidad en la parcela con tres años de descanso se debe posiblemente a la alta cobertura de teocintle, presente de manera natural durante la temporada lluviosa, que aporta residuos vegetales al suelo (Johnston-Monje y Raizada 2011), algunos de ellos fijadores de nitrógeno, como Azospirillum y Klebsiella (Carcaño-Montiel et al. 2006). Esto se refuerza con el hecho de que los exudados producidos por las raíces de maíz y teocintle contribuyen a mejorar la microbiota del suelo y algunos de los microbios endófitos presentes en sus raíces son capaces de emigrar hacia la rizosfera circundante y colonizarla, lo que sugiere un aporte directo de microorganismos al suelo (Johnston-Monje y Raizada 2011).

\section{CONCLUSIONES}

Se confirmó la resiliencia de las comunidades microbianas del suelo en el sistema de RTQ. Se encontraron niveles similares de diversidad funcional $\left(H^{\prime}\right)$ en el suelo de parcelas con uno, tres, seis años de descanso y la biodiversidad fue menor en la parcela con más de 30 años de descanso, lo que indica estabilización basal de los microorganismos cuando el suelo no se maneja durante mucho tiempo. Las propiedades físicas del suelo no se modificaron con el tiempo de descanso de las parcelas, pero el $\mathrm{pH}$, el contenido de $\mathrm{MO}$ y el de nitratos si se modificó; teniendo los valores más altos la parcela con más de 30 años de descanso.

\section{LITERATURA CITADA}

Alegre JC, Cassel DK (1996) Dynamics of soil physical properties under alternative systems to slash-and-burn. Agriculture, Ecosystems \& Environment 58: 39-48.

Amorim LB, Salcedo IH, Pareyn FGC, Alvarez IA (2014) Assessment of nutrient returns in a tropical dry forest after clear-cut without burning. Nutrient Cycling in Agroecosystems 100: 333-343.

Arteaga-Garibay RI, Gómez-Estrada MM, Martínez-Peña MD, Cadena-Zamudio JD (2016) Diversidad metabólica funcional de comunidades microbianas asociadas a suelo rizosférico de maíz (Zea mays L.) razas Amarillozamorano y Jala. Agroproductividad 9: 87-91.

Arteta R (2001) Comparación de métodos de análisis mecánico de suelos. Terra Latinoamericana 19: 219-225.

Baethgen WE, Alley MM (2008) A manual colorimetric procedure for measuring ammonium nitrogen in soil and plant Kjeldahl digests. Communications in Soil Science and Plant Analysis 20: 961-969.

Bastida F, Zsolnay A, Hernández T, García C (2008) Past, present and future of soil quality indices: a biological perspective. Geoderma 147: 159-171.

Bray RH, Kurtz LT (1945) Determination of total, organic, and available forms of phosphorous in soils. Soil Science 59: 39-46.

Carcaño-Montiel MG, Ferrera-Cerrato R, Pérez-Moreno J, Molina-Galán JD, Yoav B (2006) Actividad nitrogenasa, producción de fitohormonas, sideróforos y antibiosis en cepas de Azospirillum y Klebsiella aisladas de maíz y teocintle. Terra Latinoamericana 24: 493-502. 
Castellanos J, Jaramillo VJ, Sanford Jr RL, Kauffman JB (2001) Slash-and-burn effects on fine root biomass and productivity in a tropical dry forest ecosystem in Mexico. Forest Ecology and Management 148: 41-50.

Cochet $\mathrm{H}$ (2001) Agricultura de tumba y quema, ganadería extensiva y degradación ambiental en la Sierra Madre del Sur (Sierra de Coalcomán, Michoacán). In: Hernández L (ed.) Historia ambiental de la ganadería en México. Instituto de Ecología. México. pp. 114-122.

Di Ciocco CA, Sandler RV, Falco LB, Coviella CE (2014) Actividad microbiológica de un suelo sometido a distintos usos y su relación con variables físico- químicas. Revista de la Facultad de Ciencias Agrarias 46: 73-85.

Doran JW, Zeiss MR (2000) Soil health and sustainability: managing the biotic component of soil quality. Applied Soil Ecology 15: 3-11.

Dos Santos JCN, De Andrade EM, Medeiros PHA, Guerreiro MJS, Palácio HAQ (2017) Effect of rainfall characteristics on runoff and water erosion for different land uses in a tropical semiarid region. Water Resources Management 31: 173-185.

Fernández C, Fontúrbel T, Vega JA (2019) Effects of pre-fire site preparation and post-fire erosion barriers on soil erosion after a wildfire in NW Spain. Catena 172: 691-698.

Ferreras L, Toresani S, Bonel B, Fernández E, Bacigaluppo S, Faggioli V, Beltrán C (2009) Parámetros químicos y biológicos como indicadores de calidad del suelo en diferentes manejos. Suelo 27: 103-114.

Fregoso TLE (2008) Cambios en las características químicas y microbiológicas de un vertisol inducidos por sistemas de labranza de conservación. Terra Latinoamericana 26: 161-170.

García E (2004) Modificaciones al sistema de clasificación climática de Köppen. 5a Edición. Instituto de Geografía - Universidad Nacional Autónoma de México. México, 90p.

Garland JL, Mills AL (1991) Classification and characterization of heterotrophic microbial communities on the basis of pattern of community-level sole-carbon-source utilization. Applied and environmental microbiology 57: 2351-2359.

Giardina CP, Sandford Jr. RL, Dockersmith IC (2000a) Changes in soil phosphorus and nitrogen during slashand-burn clearing of a dry tropical forest. Soil Science Society of America Journal 64: 399-405.

Giardina CP, Sanford Jr. RL, Dockersmith IC, Jaramillo VJ (2000b) The effects of slash burning on ecosystem nutrients during the land preparation phase of shifting cultivation. Plant and Soil 220: 247-260.

Ginebra AM, Rodríguez AM, Calero MB, Ponce de León D, Font VL (2015) Carbono lábil como un indicador de cambios en dos suelos bajo diferentes usos. Cultivos Tropicales 36: 64-70.

Gómez-Vasconcelos MG, Oros PU, Garduño MVH, Hernandez-Madrigal VM (2015) La Caldera de Villa Madero: vulcanismo explosivo del cuaternario en el borde sureste del campo volcánico Michoacán-Guanajuato Martha. Ciencia Nicolaita 65: 12-35.

Jackson LE, Calderón FJ, Steenwerth KL, Scow KM, Rolston DE (2003) Responses of soil microbial processes and community structure to tillage events and implications for soil quality. Geoderma 114: 305-317.

Jaurixje M, Torres D, Mendoza B, Henríquez M, Contreras J (2013) Propiedades físicas y químicas del suelo y su relación con la actividad biológica bajo diferentes manejos en la zona de Quíbor, Estado Lara. Bioagro 25: 47-56.

Johnston-Monje D, Raizada MN (2011) Conservation and diversity of seed associated endophytes in Zea across boundaries of evolution, ethnography and ecology. Plos one 6: 1-22. 
Kaffe-Abramovich T, Steinberger Y (2006) Soil microbial functional diversity response following nematocide and biocide amendments in a desert ecosystem. Soil Biology and Biochemistry 38: 1966-1976.

Karlen DL, Mausbach MJ, Doran JW, Cline RG, Harris RF, Schuman SE (1997) Soil Quality: a concept, definition, and framework for evaluation. Soil Science Society of America Journal 61: 4-10.

Kotto-Same J, Woomer PL, Appolinaire M, Louis Z (1997) Carbon dynamics in slash-and-bum agriculture and land use alternatives of the humid forest zone in Cameroon. Agriculture, Ecosystems \& Environment 809: 245-256.

Kumar U, Shahid M, Tripathi R, Mohanty S, Kumar A, Bhattacharyya P, et al. (2017) Variation of functional diversity of soil microbial community in sub-humid tropical rice-rice cropping system under long-term organic and inorganic fertilization. Ecological Indicators 73: 536-543.

Li, Y, Q Zhang, Y Cai, Q Yang SX Chang (2020) Minimum tillage and residue retention increase soil microbial population size and diversity: Implications for conservation tillage. 716: 137164. Doi: 10.1016/j.scitotenv.2020. 137164

Maass JM, Balvanera P, Castillo A, Daily GC, Mooney HA, Ehrlich P, et al. (2005) Ecosystem services of tropical dry forests: insights from long- term ecological and social research on the Pacific Coast of Mexico. Ecology and Society 10: 17.

Manjunath M, Kumar U, Yadava RB, Rai AB, Singh B (2018) Influence of organic and inorganic sources of nutrients on the functional diversity of microbial communities in the vegetable cropping system of the IndoGangetic plains. Comptes Rendus - Biologies. Academie des sciences 341: 349-357.

Mataix-Solera J, Cerda A, Arcenegui V, Jordán A, Zavala LM (2011) Fire effects on soil aggregation: a review. Earth-Science Reviews 109: 44-60.

Medeiros EV, Duda GP, Dos Santos LAR, Lima JR de S, De Almeida-Cortêz JS, Hammecker C, Lardy L, Cournac $L$ (2017) Soil organic carbon, microbial biomass and enzyme activities responses to natural regeneration in a tropical dry region in Northeast Brazil. Catena 151: 137-146.

Moya D, González-De Vega S, García-Orenes F, Morugán-Coronado A, Arcenegui V, Mataix-Solera J, LucasBorja ME, De Las Heras J (2018) Temporal characterization of soil-plant natural recovery related to fire severity in burned Pinus halepensis Mill. forests. Science of the Total Environment 640-641: 42-51.

Ochoa V, Hinojosa B, Gómez-Muñoz B, García-Ruiz R (2007) Actividades enzimáticas como indicadores de calidad del suelo en agroecosistemas ecológicos. Revista Electrónica Universidad de Jaen 2: 1-10.

Pagano MC, Schalamuk, Cabello MN (2011) Arbuscular mycorrhizal parameters and indicators of soil health and functioning: applications in agricultural and agroforestal systems. In: Miransari M. (ed.). Soil microbes and environmental health. Edition: 1. Nova Science Publishers. New York. pp. 267-276.

Pérez-García O, Del Castillo RF (2016) The decline of the itinerant milpa and the maintenance of traditional agrobiodiversity: Crops and weeds coexistence in a tropical cloud forest area in Oaxaca, Mexico. Agriculture, Ecosystems and Environment 228: 30-37.

Revillini D, Gehring CA, Johnson NC (2016) The role of locally adapted mycorrhizas and rhizobacteria in plant-soil feedback systems. Functional Ecology 30: 1086-1098.

Riveiro-Filho AA, Adams C, Murrieta RSS (2013) The impacts of shifting cultivation on tropical forest soil: a review. Boletim do Museu Paraense Emílio Goeldi. Ciências Humanas 8: 693-727.

Rodrigues EL, Jacobi CM, Figueira JEC (2019) Wildfires and their impact on the water supply of a large neotropical metropolis: a simulation approach. Science of the Total Environment 651: 1261-1271. 
Rojas RT (1991) La agricultura en tierras mexicanas, desde sus orígenes hasta nuestros días. Grijalbo. Consejo Nacional para la Cultura y las Artes. México. 420p.

Rzedowski J (2006) Vegetación de México. Comisión Nacional para el Conocimiento y Uso de la Biodiversidad. México. 505p.

Sharmah D, Jha DK (2014) Diversity of arbuscular mycorrhizal fungi in undisturbed forest, slash-and-burn field, and monoculture forest of Indo-Burma megadiverse region. Revista Brasileira de Botanica 37: 339-351.

Singh AK, Kushwaha M, Rai A, Singh N (2017) Changes in soil microbial response across year following a wildfire in tropical dry forest. Forest Ecology and Management 391: 458-468.

Singh JP (1988) A rapid method for determination of nitrate in soil and plant extracts. Plant and Soil 110: 137-139.

Smith AP, Marín-Spiotta E, Balser T (2015) Successional and seasonal variations in soil and litter microbial community structure and function during tropical postagricultural forest regeneration: a multiyear study. Global Change Biology 21: 3532-3547.

Socarrás A (2013) Mesofauna edáfica: indicador biológico de la calidad del suelo. Pastos y Forrajes 36: 5-13.

Spohn M (2016) Element cycling as driven by stoichiometric homeostasis of soil microorganisms. Basic and Applied Ecology 17: 471-478.

Toledo VM, Barrera-Bassols N, García-Frapolli E, Alarcón-Chaires P (2008) Uso múltiple y biodiversidad entre los mayas Yucatecos (México). Interciencia 33: 345-352.

Tomson P, Bunce RGH, Sepp K (2016) Historical development of forest patterns in former slash and burn sites in sourthern Estonia. In: Agnoletti M, Emanueli F (Eds). Biocultural Diversity in Europe. Environmental History 5: 303-318.

Urretavizcaya M (2010) Propiedades del suelo en bosques quemados de Austrocedrus chilensis en Patagonia, Argentina. Bosque 31: 140-149.

Wilson C, Kampf SK, Wagenbrenner JW, MacDonald LH (2018) Rainfall thresholds for post-fire runoff and sediment delivery from plot to watershed scales. Forest Ecology and Management 430: 346-356.

WingChing-Jones R, Uribe LL (2016) Biomasa y actividad microbiana en suelos de uso ganadero y en regeneración de bosque. Cuadernos de Investigación UNED 8: 107-113.

Zamora A, Malaver N, Ramos J (2012) Análisis funcional de microorganismos: un estimador de diversidad y estructura comunitaria. Acta Biológica Venezolana 32: 57-86. 